\title{
Dynamic analysis of emergency inter-organizational communication network under public health emergency: a case study of COVID-19 in Hubei Province of China
}

\author{
Yunmeng $\mathrm{Lu}^{1} \cdot$ Tiezhong Liu $^{1} \cdot$ Tiantian Wang $^{1}$
}

Received: 24 March 2021 / Accepted: 28 June 2021 / Published online: 6 July 2021

(c) The Author(s), under exclusive licence to Springer Nature B.V. 2021

\begin{abstract}
Public health emergencies, especially major infectious diseases, may cause global crises. Timely and effective communication is essential for response to such incidents. However, the emergency response to such incidents usually lasts longer and break out repeatedly, and the existing static emergency communication network (ECN) analysis cannot fully reflect the dynamic information interaction between organizations during the emergency process. Therefore, this article takes the recent COVID-19 epidemic in Hubei, China as a case, and uses social network analysis to reveal the dynamic evolution of communication networks, positions, roles, and tasks of organizations from the time dimension. The results show that: (1) the ECN has changed from concentrated to decentralized over time; (2) the positions and roles of participating organizations in the ECN has changed, but there are still a few key organizations that at the central position in all phases of emergency communication; (3) the core tasks have changed due to emergency needs at each stage; (4) under the concentrated management system, the core organization of the ECN mainly comes from government organizations. The research results reveal the dynamic evolution of communication networks between different types of emergency organizations, which is beneficial to guide emergency management of public health emergencies. In actual emergency, the emergency communication mode should be dynamically adjusted based on the characteristics of the emergency situation at different stages, comprehensively using the advantages of the concentrated and decentralized emergency network. In addition, communication between different types of organizations such as governments, research institutions, and enterprises should be strengthened, and channels for diversified organizations to participate in emergency communication should be set up. The research helps to improve communication between emergency response organizations and is of great significance to controlling and reducing the harm caused by public health emergencies.
\end{abstract}

Keywords Emergency communication network · Public health emergencies · Dynamic evolution $\cdot$ COVID-19 emergency $\cdot$ Network analysis

Tiezhong Liu

3120185815@bit.edu.cn

1 School of Management and Economics, Beijing Institute of Technology, No. 5, South Street, Zhongguancun, Haidian District, Beijing, China 


\section{Introduction}

In the emergency management of public health emergencies, due to the limitations of the public health department in terms of manpower, resources, information, etc., it is necessary to rely on the coordinated response of government organizations (GOs) and non-governmental organizations (NGOs) to avoid the collapse of the entire system due to the fragility of the emergency system. In particular, major infectious diseases are likely to cause widespread epidemics, such as the spread of the COVID-19 worldwide (Adiyoso 2020; Lim and Nakazato 2020; Liu et al. 2020). In December 2019, the disease was reported for the first time by Wuhan (CDC 2020), and was declared by World Health Organization (WHO) as a "Public Health Emergencies of International Concern" (PHEIC) on January 30, 2020. On March 11, the WHO announced that the COVID-19 can be characterized as a global pandemic (WHO 2020b). As of February 4, 2021, the COVID-19 has caused 103,631,793 infections and 2,251,613 deaths worldwide according to the situation reports by WHO (WHO 2020a). Major epidemics are characterized by risk uncertainty, multiple subjects, risk sharing, and huge damage, which challenge the country's emergency response capabilities. Due to the high degree of uncertainty in the virus characteristics and response measures in the epidemic, it requires timely and sensitive information communication between emergency organizations to promote the rational flow and optimal allocation of resources, and then make effective emergency response.

The emergency evolution of public health emergencies is actually a series of effects of the spread of emergency information (Wen et al. 2011). Emergency information exists in the emergency collaboration network, and efficient communication between organizations is a key step to support their emergency collaboration, and it is vital to the implementation of emergency operations(Clark-Ginsberg 2020; Opdyke et al. 2017). However, there are still some challenges in the current emergency work, such as insufficient communication and collaboration (Pasman and Suter 2005). Therefore, some scholars began to pay attention to inter-organizational communication and collaboration during emergency response. For example, Harris and Doerfel (2017) analyzed the emergency cooperation relationship between organizations in the emergency response under the superstorm Sandy and explained that it played an important role in emergency response and disaster recovery. Rajput et al. (2020) analyzed the information communication between GOs and NGOs on social media under Hurricane Harvey in Houston, revealing the role of organizations in disasters. Jung and Park (2016) analyzed the inter-organizational information network in emergency response, revealing the sufficient information flow between organizations to promote effective collaboration between organizations to respond to emergencies. In addition, there are many uncertain factors in the communication process of emergency information, such as the changes in external environment, emergency organizations and their interaction, and the timeliness of emergency information. The social network analysis (SNA) is an effective method to analyze the complexity and uncertainty of an event (Comfort et al. 2012). This method graphically shows the relationship between emergency collaboration and information interaction between organizations, and can be used to analyze and explain the communication between emergency organizations (Hu and Kapucu 2016). Through networked analysis, it can be used to guide more effective actions to adapt to changing emergency situations (Alkhatib et al. 2019; Kapucu and Hu 2016).

Researchers have used SNA to carry out a series of studies on organizational collaboration and communication in emergency response in different fields. Du et al. (2020) has built a collaborative network to deal with hazardous chemical accidents, emphasizing the need 
to strengthen collaboration among public sector organizations, non-profit organizations (NPOs) and private organizations (POs). Curtis (2018) studied the emergency response network after Hurricane Katrina and revealed that isolated or peripheral organizations hindered effective response. Kapucu and Garayev (2016) compared the vertical and horizontal emergency networks of two counties in Florida, which found that the horizontal network is more conducive to effective emergency response. In addition, some research focuses on using node centrality measures to identify important participants in the emergency network (Hu et al. 2014; Koliba et al. 2018). Guo and Kapucu (2015) identified the key emergency actor by analyzing the emergency cooperation network of China's low temperature freezing rain and snow disasters. Tang et al. (2017) analyzed China's nuclear power plant accident emergency network from both the micro and macro levels, and found that the public sector occupies an important position in the emergency. Kim et al. (2013) discussed the status of participants in disaster response networks.

Although the emergency response researches are based on the static snapshots of interorganizational interaction provided by SNA, which clearly illustrates the composition and structure of the network, the dynamic characteristics of the emergency process are not reflected. In recent years, some studies have begun to focus on the dynamics of the emergency process (Abbasi and Kapucu 2012). In order to clarify the evolution mechanism of the emergency cooperation network, Abbasi (2014) explored the formation of connections between participants in the emergency cooperation network. Abbasi et al. (2018) explored the temporal dynamics of Australia's extreme bushfire response network structure, and found that the response network is gradually decentralized. However, few studies have analyzed the dynamics of the emergency coordination network for public health emergencies. As for the emergency response to public health emergencies, it involves the participation of many different types of organizations, and the participants and their roles continue to change with the evolution of the event. At the same time, as emergency information flows through the network, organization joining, exiting, location changes, as well as the interruption of a contact and the establishment of new contacts will have an important impact on emergency communication between organizations. Therefore, in the rapidly changing emergency environment of public health emergencies, it is necessary to analyze the dynamic evolution of its communication network and pay attention to how the communication network changes over time, so as to effectively guide actual emergency activities.

The COVID-19 epidemic is a major public health emergency with the fastest spread, the widest range of infections, and the most difficult prevention and control since the founding of China. In order to prevent the spread of the COVID-19 epidemic, Hubei, China, was the first to adopt prevention and control measures such as a lockdown strategy on Wuhan. And as the understanding of the epidemic has gradually deepened, a reasonable emergency management mechanism for joint prevention and control has been formed. In this process, there are many points worthy of reflection and reference for emergency management and communication. In addition, the emergency network is related to the inter-organizational relationship in the management system. Hteina et al. (2018) studied Myanmar floods emergency response network, and found that changes in the regime may affected the organization's cooperation mode during the emergency process. And the inter-organizational relationship between decentralized management and centralized management system are significant different to each other (Guo and Kapucu 2015). But most researches has focused on emergency response under the decentralized management system (Abbasi and Kapucu 2012; Abbasi et al. 2018). Therefore, this research focuses on the COVID-19 Emergency Inter-organizational Communication Network (COVID-19-EIOCN) in Hubei, China from the perspective of time dynamics. Specifically, this article attempts to answer the following 
questions: (1) How does COVID-19-EIOCN evolve over time? (2) How does the position/ role of an organization in the network changes in different stages? (3) How does the relationship between emergency organizations and their communication tasks change in different stages of COVID-19 emergency?

\section{Data and method}

\subsection{Study design}

In the emergency process of public health emergencies, participating organizations need to conduct timely and effective communication. In order to comprehensively analyze the communication and its dynamic evolution among emergency organizations of such uncertain events, we designed the research framework shown in Fig. 1. The research framework includes three steps: (1) Data collection and coding, to extract various organizations participating in the COVID-19 emergency and their communication relationships, and tasks supported by emergency communications. First, identify the emergency organizations and their interactions from the epidemic prevention and control situation reported by official government websites and news reports. Then, according to the actual emergency activities and emergency plans of public health emergencies, the emergency inter-organizational communication support tasks (EIOCSTs) was determined. (2) Build networks. Based on the development of COVID-19, the emergency interactions are divided into three stages by time slicing, thereby obtaining the communication relationship matrix of each stage, and constructing a visual network topology diagram, where nodes represent organizations, colors represent organization types, and lines represent communication relationships between emergency organizations. (3) Conduct emergency communication network (ECN) analysis. First, analyze the dynamic evolution of the network through indicators such as network density, average path length and network centrality. Secondly, using node degree centrality and betweenness centrality to examine the changes of positions and roles of

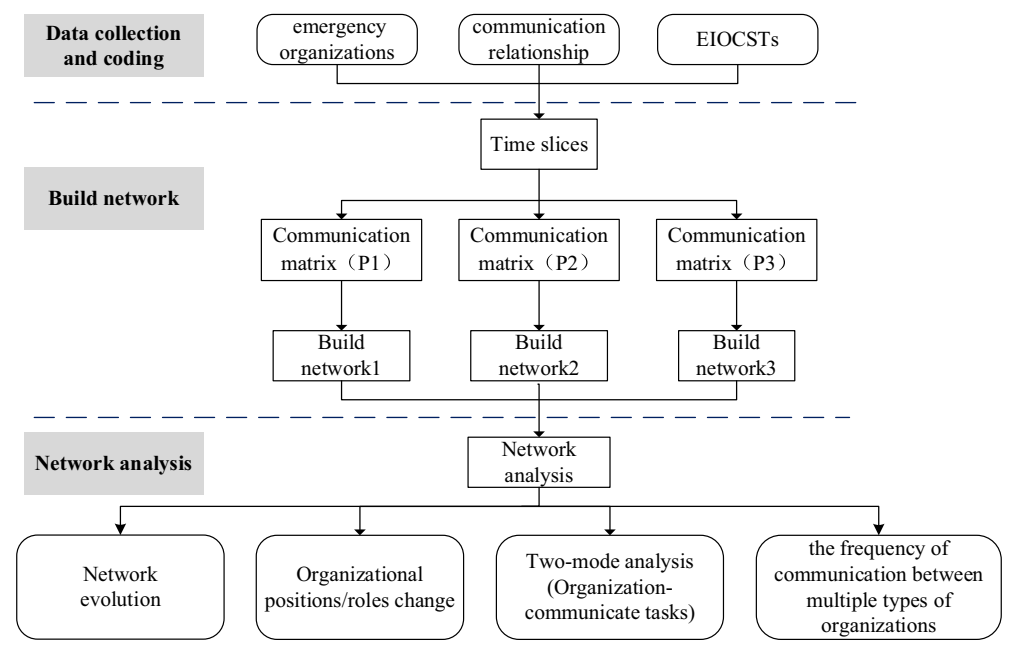

Fig. 1 Research framework 
organizations in the network. Then, according to the relationship between the organization and communication tasks, a Two-mode network is constructed to identify key communication tasks at different stages and analyze the evolution of the organization's emergency communication tasks. Finally, the frequency of communication between different types of organizations in the entire network is discussed.

\subsection{Case background}

In December 2019, cases of COVID-19 were detected in Wuhan, Hubei Province, and some public health departments conducted etiological and epidemiological investigations. On January 23, 2020, the Chinese government implemented a lockdown strategy on Wuhan to control and prevent the further spread of the epidemic. Subsequently, Hubei Province raised the emergency level of COVID-19 to the highest emergency state (firstlevel response). On February 21, 2020, Effective progress was made in curbing the spread of the virus. According to the development of the epidemic prevention and control situation, the Central Committee of the Communist Party of China (CCCPC) made the decision to coordinate the epidemic prevention and control and economic development, and began to resume work and production orderly. The case we selected in the study because:

1. COVID-19 is considered to be the most widespread global pandemic. $95.44 \%$ of the confirmed cases in China are concentrated in Wuhan, Hubei Province (updated to March 17, 2020). Hubei COVID-19 emergency communication research can reflect China's emergency response to the epidemic, which is representative.

2. The effect of non-pharmaceutical interventions implemented in Wuhan is obvious, which may reduce the number of COVID-19 infections by 67 times (Lai et al. 2020). These non-pharmaceutical interventions require different types of organizations to conduct emergency interaction around the core tasks of "monitoring and early warning, comprehensive coordination and medical rescue, etc.".

3. Due to the long duration of the COVID-19 epidemic and its emergency, the participating organizations and their main tasks in emergency communication change dynamically over time.

\subsection{Data collection}

This sub-section collects and extracts the data on the prevention and control of the CIOVD19 epidemic on government official websites and news reports. These data are derived from the epidemic prevention and control situation and official documents published on the official websites of Government of the People's Republic of China (GPRC), National Health Commission of the People's Republic of China (NHCPRC), the Government of Hubei Province (GHP) and Health Commission of Hubei Provincial (HCHP), including Hubei Province's situation update of COVID-19, Hubei Province epidemic prevention and control notice and emergency plan for public health emergencies in Hubei Province, etc., which records the emergency interactions between organizations in detail. In addition, news reports (China News, People's Daily) provide multi-source data on interactions between organizations in epidemic prevention and control (Abbasi et al. 2018).

We comprehensively use the methods of manually checking websites and compiling ptyhon codes to crawl webpage list data to obtain the texts in the above mentioned official website documents from December 27, 2019 to March 17, 2020, numbered and classified 
all participating organizations, including the names and types of emergency organizations, and recorded the communication relationships and communication time. First, by analyzing the content of the text, find out the emergency organization involved in the epidemic prevention and control from the text information of the government official documents. For example, the participation of emergency organizations such as NHCPRC, Ministry of Public Security of the People's Republic of China (MPSPRC), and Ministry of Transport of the People's Republic of China (MTPRC) can be obtained from the prevention and control dynamics on the official website of the NHCPRC (http://www.nhc.gov.cn/xcs/fkdt/202001/ 5701f2f6177345ffa0b9f0421bd4034d.shtml). Second, extract the interactive relationship between emergency organizations. In an emergency response, participating organizations need to communicate to share rescue information, command transmission, task collaboration, or meeting interaction. If the organization participates in any of the above activities, it indicates that there is an interactive relationship between the organizations. Therefore, we use the method of manually interpreting the text content to extract the communication relationship between organizations, where the time involved in the text or the signing time of the information release is designated as the interaction time. For example, according to the epidemic prevention and control work dynamics of NHCPRC, six departments including the NHCPRC, MPSPRC, MTPRC, General Administration of Customs of the People's Republic of China (GACPRC), Civil Aviation Administration of China (CAAC), and China State Railway Group Co.,Ltd. (CSRG) issued the "Notice on Strictly Preventing Pneumonia Caused by the Transmission of COVID-19 Through Transportation", we extract that there is interaction between NHCPRC and MPSPRC, GACPRC, etc. In addition, comprehensively considering the functions of emergency organizations in public health incidents and their forms of participation, we uniquely identify the type of organization based on the most relevant principles. Therefore, we have identified 137 organizations and 472 links, including 38 national government organizations (NAGOs), 56 provincial government organizations (PGOs), and 20 municipal government organizations (MGOs), 8 research institutions (RIs), 9 POs, 3 NPOs and 3 international organizations (IOs).

The emergency plan for public health emergencies is a key guideline for emergency response, which is the most authoritative source for determining emergency communication tasks. So, the EIOCSTs are mainly derived from emergency plans for public health emergencies in Hubei Province (http://www.hubei.gov.cn/zwgk/yjgl/yjya/201105/t2011 0510_243414_1.shtml). Finally, we have compiled 11 EIOCSTs, as shown in Table 1.

Table 1 Emergency interorganizational communication support tasks (EIOCSTs)

\begin{tabular}{ll}
\hline Number & Emergency communication support tasks \\
\hline EIOCST1 & Logistic Support \\
EIOCST2 & Monitoring and Warning \\
EIOCST3 & Communications and Transportation \\
EIOCST4 & Medical treatment and Epidemic prevention \\
EIOCST5 & Material Support \\
EIOCST6 & Order Maintenance \\
EIOCST7 & Comprehensive Coordination \\
EIOCST8 & Technology Support \\
EIOCST9 & Information Release \\
EIOCST10 & Security Maintenance \\
EIOCST11 & Work Resumption \\
\hline
\end{tabular}




\subsection{Network analysis of COVID-19-EIOCN}

SNA can reveal the evolution of communication relationships between emergency organizations, which can provide visual analysis (Howes et al. 2015; Provan and Lemaire 2012). Measuring the relationship between nodes and identifying key nodes is an important part of SNA (Scott 2013). The COVID-19 emergency requires flexible and dynamic communication and collaboration between organizations to implement non-pharmacological interventions or rescue operations. Based on the above COVID-19 data, we transformed the inter-organizational interactions into an adjacency matrix and constructed the COVID19-EIOCN, as shown in Fig. 2. The study measures the dynamic characteristics of COVID19-EIOCN through the density, network centrality and node centrality indicators to effectively analyze the evolution of the COVID-19-EIOCN and the positions and tasks of organizations.

\subsubsection{Network structure analysis}

The Network structure analysis of this study mainly examines the comprehensive structure and closeness of COVID-19-EIOCN through the network density and network centralization. Network density and network centralization reflect different levels of network compactness. Density represents the cohesion of the network, and network centralization explains the degree of this cohesion around a specific node (Scott 2017).

2.4.1.1 Density Density is the ratio between the number of links actually established in the network and the total number of links that can theoretically exist at most. The calculation formula for the theoretical maximum number of links is $n(n-1) / 2$, where $n$ is the number of nodes. Density measures the level of interconnection among all participants in the network

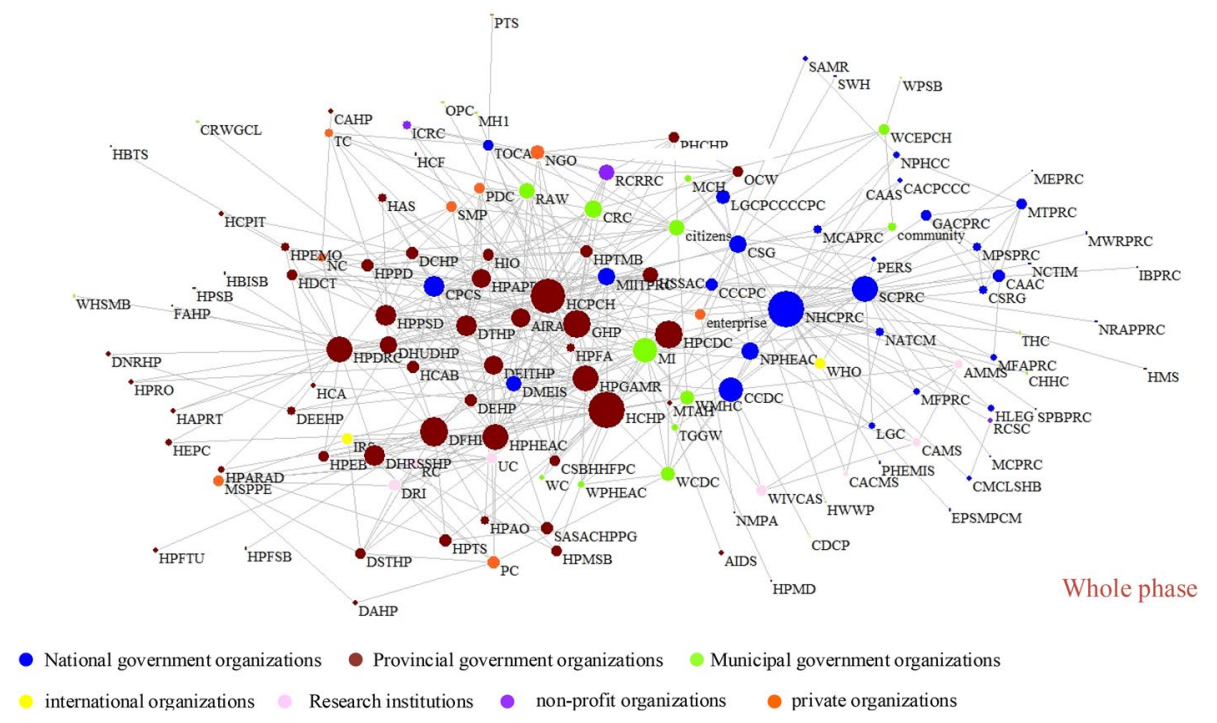

Fig. 2 The whole emergency inter-organizational communication network of COVID-19. Note: The size of nodes set by degree centrality. The Organization's full names in Fig. 2 are listed in Table 6 of Appendix 1 
and reflects the cohesion of the network (Abbasi et al. 2018). The reliability and speed of emergency information flow between nodes are critical to emergency response (Wolbers et al. 2013). In a denser network, the higher the connectivity of information exchange between members, efficient information interaction helps to improve the operating efficiency of the network (Abbasi and Kapucu 2016), but their establishment and maintenance costs are very high.

2.4.1.2 Network centralization Network centrality refers to the degree of centralization of the entire network, which can be used to determine whether the network has a centralized structure. The centrality of the network reveals the degree of difference in the centrality of each member (Abbasi and Kapucu 2012). The calculation method of which is to evaluate the difference between the centrality score of the most central node and the centrality scores of other nodes, and calculate the ratio of the sum of these differences to the sum of the largest possible differences. In a high-centered network, emergency communication is mainly concentrated on a small number of members, which may cause the paralysis of information dissemination between organizations due to information overload.

\subsubsection{Node centrality analysis}

Evaluating the centrality of nodes in the network helps us understand the role and position of organizations in emergency situations (Rajput et al. 2020). In the COVID-19-EIOCN node centrality assessment, we mainly examined two attributes: Degree centrality and Betweenness centrality, revealing the importance of each organization's role and location in the network (Kim and Hastak 2018).

2.4.2.1 Degree centrality Degree centrality is measured by the number of direct connections a node has in the network (Freeman 1978), which can identify participants with extensive direct interaction. In the ECN, organizations with a high degree of centrality may have access to more information and resources (Liu et al. 2020), and they are usually regarded as emergency information hubs in actual emergency scenarios.

2.4.2.2 Betweenness centrality Betweenness centrality refers to the degree to which a node is located on the shortest path between each pair of nodes in the network (Robinson 2012), it measures the degree of a node's monopoly on information between nodes. In the ECN, participants with greater betweenness centrality play the role of information bridge, occupying the main path of information flow, so they have more opportunities to control and guide the information flow between participants.

\section{Results}

This section explores the actual interactions during the COVID-19 emergency in different phases. According to the evolution of Wuhan COVID-19 and related literature, we sliced its emergency response process into three phases: (1) Phase P1: From December 27, 2019 to January 20, 2020, cases of COVID-19 were detected in Wuhan. (2) Phase P2: From January 20, 2020 to February 21, 2020, the newly confirmed cases increased rapidly, and the 
prevention and control situation was extremely severe. Wuhan Implement a city lockdown strategy. (3) Phase P3: From February 21, 2020 to March 17, 2020, the spread of the virus had been contained.

\subsection{The evolution of COVID-19-EIOCN}

To explore the evolution of COVID-19-EIOCN, this study uses Pajek to measure the structural characteristics of COVID-19-EIOCN at different stages. Table 2 shows the measurement indicators for the whole process and three phases, including the number of organizations (nodes), the frequency of inter-organizational communication (links), network density, and network centrality.

As shown in Table 2, from Dec 27, 2019 to March 17, 2020, the constructed COVID19-EIOCN consists of 137 emergency organizations, and 472 links have been established between them. The measured density and centralization of the network are 5.07 and $27.69 \%$, respectively, which indicates that the communication network in the whole process is sparse. The average path length of the network is 2.81 , indicating that on average, about 3 intermediaries are needed between organizations to get contact.

In addition, the statistical indicators of P1, P2, and P3 show that COVID-19-EIOCN has evolved from concentrated to decentralized. Firstly, the network densities of P1 to P3 are $17.89 \%, 7.05 \%$, and $5.2 \%$, respectively, showing a gradual decline. It shows that the network in phase P1 is the closest, and then becomes sparse. Secondly, the network centralization and density have the same trend. The network centralization in phase P1 is the highest, at $61.99 \%$, indicating that the network is concentrated in one or several organizations. To a certain extent, the communication between emergency organizations depends on a small number of organizations. In phases P2 and P3, the centralization of the network gradually declines, indicating that the information and power of the organizations in COVID19 -EIOCN are gradually being dispersed, and the network have changed from centralized to decentralized. Thirdly, the number of organizations and communication relationships are increasing first and then decreasing. In phase P1, the number of organizations is the least (20), and the communication relationships between organizations is 34 . In phase P2, the number of organizations and interactions is the largest, with 356 communication relationships among 101 organizations. Finally, the average path length of the three phases gradually increases, which indicates that the average number of intermediaries that organizations need to pass through to get in touch with each other has increased. The average path of phase P3 is 3.24, which is the longest, indicating that the relationship between organizations at this phase is relatively sparse.

Table 2 The attributes of COVID-19-EIOCN over time

\begin{tabular}{lllll}
\hline & Whole process & P1 & P2 & P3 \\
\hline \# of Organizations (nodes) & 137 & 20 & 101 & 69 \\
\# of Interactions (links) & 472 & 34 & 356 & 122 \\
Density (\%) & 5.07 & 17.89 & 7.05 & 5.2 \\
Network Centralization (\%) & 27.69 & 61.99 & 28.52 & 26.45 \\
\# of Components (sub-networks) & 1 & 1 & 1 & 1 \\
Average path length & 2.81 & 2.12 & 2.8 & 3.24 \\
\hline
\end{tabular}




\subsection{Dynamic analysis of emergency organization positions}

This section analyzes the degree centrality and betweenness centrality of organizations in COVID-19-EIOCN at different phases to identify the key organizations and reveal the dynamic changes in the positions of organizations. Figure 3 visualizes the COVID19-EIOCN and its node degree centrality in P1, P2 and P3.

The color and size of the nodes in Figs. 2 and 3 indicate the categories and positions of the organization. In the whole phase communication network, Hubei COVID19 Prevention and Control Headquarters $(\mathrm{HCPCH})$, NHCPRC, GHP, HCHP and Hubei Provincial Development and Reform Commission (HPDRC) are the five most influential organizations, which are both national and provincial government agencies. From P1 to P3, the key organizations at each phase change with the development of the network. In phase P1, NHCPRC, Chinese Center for Disease Prevention and Control (CCDC), Wuhan Municipal Health Commission (WMHC), Medical Institution (MI) and HCHP are active participant, which occupy central positions. It is worth noting that the organizations involved in phase $\mathrm{P} 1$ are mainly health administrative departments and research institutions. In stage P2, HCPCH, GHP and Hubei Province General Administration of Market Regulation (HPGAMR) occupy the new center position of the network. They interact with private organizations, research institutions and non-profit organizations, and play an important role in resource coordination. In stage P3, the HPDRC, Department of Finance of Hubei Province (DFHP) and Department of Human Resources and Social Security of Hubei Province (DHRSSHP) have become the new key nodes of the network. Although the key emergency organizations at each phase have changed, government organizations are in a more central position compared with other types of organizations in COVID-19-EIOCN.

To accurately analyze the positions of the organizations, we analyzed the degree centrality and betweenness centrality of the COVID-19-EIOCN node in each phase. Table 3 shows the top-ten degree centrality participants. In the ECN, organizations with greater degree of centrality have more resources and information, and are more likely to generate and disseminate information. In Table 3, State Council of the PRC (SCPRC), NHCPRC and HCHP have all remained in the top 10 in the three phases, playing the role of the information hub. It is worth noting that although the HCPCH was established in Phase P2, it remained in the top 3 at Phases P2 and P3. In addition, we analyzed important organizations that only became information hubs at a certain phase. In phase P1, many of the top 10 participants are research institutions, which are Academy of Military Medical Sciences (AMMS), Chinese Academy of Medical Sciences (CAMS) and Wuhan Institute of Virology, Chinese Academy of Sciences (WIVCAS). These subjects mainly carry out etiological and epidemiological investigations. And in phase P2, most of the organizations with high degree centrality scores are provincial governments, which indicates that the communication in this phase may be centered on provincial governments. Among them, HCPCH has the highest degree centrality in the network, followed by GHP, 2 of the top 10 participants are artificial intelligence systems. These artificial intelligence systems have made great contributions to epidemic monitoring and disease diagnosis. In phase P3, as the epidemic situation is under phased control, the main task of emergency response is to coordinate epidemic prevention and control and economic development. HPDRC, DFHP and DHRSSHP have become several information centers. These results reflect that the information center at a certain phase does not maintain a fixed position in each phase, but there are several core organizations that maintain active in two or more phases. 


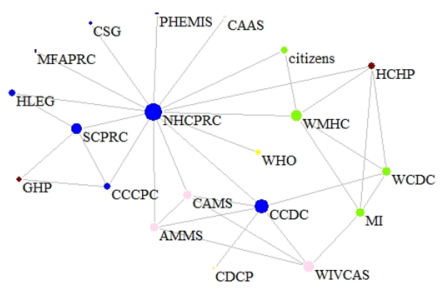

P1
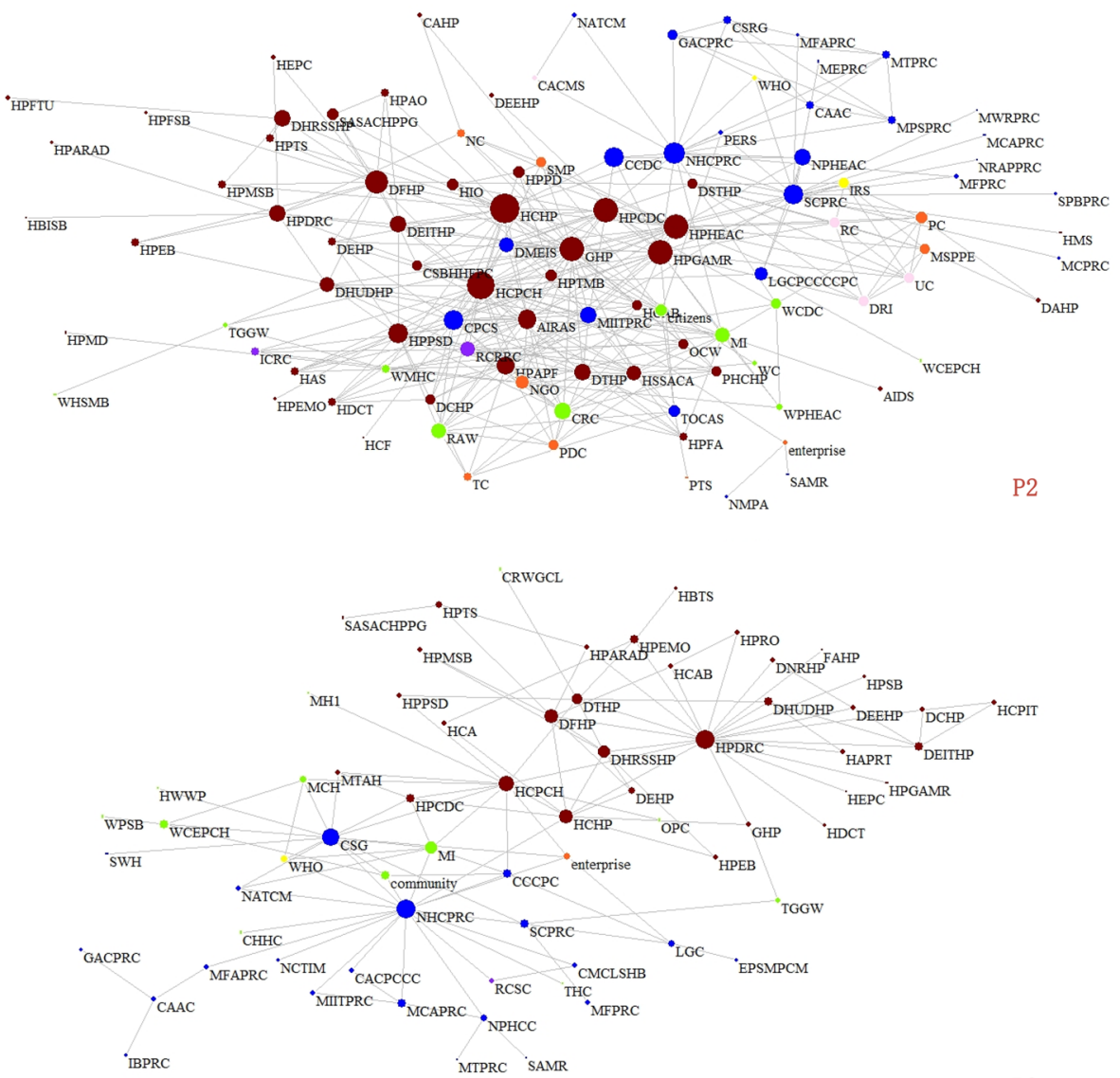

P3

- National government organizations Provincial government organizations Municipal government organizations

international organizations $\bigcirc$ Research institutions $\bigcirc$ non-profit organizations organizations

Fig. 3 Three COVID-19-EIOCN in different stages. Note: The size of nodes set by degree centrality. The Organization's full names in Fig. 3 are listed in Table 6 of Appendix 1

The betweenness centrality of a node is used to measure the extent to which an organization plays an intermediary role between each pair of nodes. Table 4 lists the top 10 organizations with high betweenness centrality in each phase. Similar to the results of degree centrality analysis, most organizations with high betweenness centrality come from government organizations, especially provincial government organizations. 
Table 3 Top 10 emergency organizations with degree centrality $(\%)$

\begin{tabular}{lllll}
\hline & Whole phase & Phase 1 & Phase 2 & Phase 3 \\
\hline 1 & HCPCH(44) & NHCPRC(14) & HCPCH(35) & HPDRC(21) \\
2 & NHCPRC(39) & CCDC(6) & GHP(29) & NHCPRC(19) \\
3 & GHP(31) & WMHC(5) & HPGAMR(22) & HCPCH(13) \\
4 & HCHP(28) & MI(4) & HCHP(22) & CSG(12) \\
5 & HPDRC(24) & HCHP(4) & HPHEAC(21) & HCHP(10) \\
6 & SCPRC(23) & SCPRC(4) & AIRAS(21) & DFHP(8) \\
7 & HPGAMR(23) & AMMS(4) & CPCS(20) & DTHP(7) \\
8 & MI(21) & WCDC(4) & SCPRC(18) & $\operatorname{DHRSSHP(7)~}$ \\
9 & HPHEAC(21) & CAMS(4) & MIITPRC(18) & $\operatorname{MI}(7)$ \\
10 & AIRAS(21) & WIVCAS(4) & NHCPRC(18) & DEITHP(5) \\
\hline
\end{tabular}

The Organization's full names in Table 3 are listed in Table 6 of Appendix 1

Table 4 Top 10 emergency organizations with betweenness centrality (\%)

\begin{tabular}{lllll}
\hline & Whole phase & P1 & P2 & P3 \\
\hline 1 & NHCPRC(28.9) & $\operatorname{NHCPRC}(77.05)$ & $\operatorname{SCPRC}(19.6)$ & NHCPRC(44.41) \\
2 & $\operatorname{HCPCH}(20.27)$ & $\operatorname{CCDC}(16.03)$ & $\operatorname{GHP}(17.47)$ & $\operatorname{HPDRC}(41.39)$ \\
3 & $\operatorname{HCHP}(13.06)$ & $\operatorname{WMHC}(6.48)$ & $\operatorname{HCPCH}(16.98)$ & $\operatorname{HCHP}(30.53)$ \\
4 & $\operatorname{SCPRC}(12.73)$ & $\operatorname{SCPRC}(5.56)$ & $\operatorname{NHCPRC}(15.95)$ & $\operatorname{HCPCH}(26.23)$ \\
5 & $\operatorname{HPDRC}(10.41)$ & $\operatorname{HCHP}(4.87)$ & $\operatorname{HCHP}(13.89)$ & $\operatorname{CSG}(13.57)$ \\
6 & $\operatorname{GHP}(9.71)$ & $\operatorname{CCCPC}(4.68)$ & $\operatorname{MiTPRC}(11.98)$ & $\operatorname{MFAPRC}(8.56)$ \\
7 & $\operatorname{HPGAMR}(9.28)$ & $\operatorname{AMMS}(2.1)$ & $\operatorname{HPGAMR}(11.77)$ & $\operatorname{DFHP}(7.16)$ \\
8 & $\operatorname{MITPRC}(6.69)$ & $\operatorname{CAMS}(2.1)$ & $\operatorname{HPHEAC}(8.93)$ & $\operatorname{NPHCC}(5.84)$ \\
9 & $\operatorname{MI}(6.26)$ & $\operatorname{WIVCAS}(1.75)$ & $\operatorname{DFHP}(7.77)$ & $\operatorname{CAAC}(5.84)$ \\
10 & $\operatorname{CSG}(5.65)$ & $\operatorname{WCDC}(1.75)$ & $\operatorname{HPDRC}(5.16)$ & $\operatorname{DTHP}(5.39)$ \\
\hline
\end{tabular}

The Organization's full names in Table 4 are listed in Table 6 of Appendix 1

In Table 4, from P1 to P3, the betweenness centrality of NHCPRC and HCHP is in the top five, have always been in bridge positions in COVID-19-EIOCN, which provide other participants with shorter communication path. Among them, NHCPRC has the highest betweenness centrality in phase P1 and P3, while the SCPRC has the highest betweenness centrality in P2 phase, they were the information broker. And, $\mathrm{HCPCH}$ entered the network in phase $\mathrm{P} 2$, and then remained in a bridge position, playing an important information broker role. In addition, research institutions have high betweenness centrality in phase P1. While HPGAMR, Ministry of Industry and Information Technology of the People's Republic of China (MIITPRC) and DFHP are important bridge organizations that play the connecting tasks in phase $\mathrm{P} 2$. In phase $\mathrm{P} 3$, resumption of work and production has become a new emergency demand, and HPDRC, DFHP and Department of transportation of Hubei Province (DTHP) have become important bridge organizations. Therefore, organizations with higher bridge capabilities in a certain phase do not remain the same in all phases, it will change with changes in emergency needs.

Based on the above analysis of COVID-19-EIOCN's node degree centrality and betweenness centrality, it is confirmed that the communication between COVID-19 
emergency organizations is a dynamic process, and the roles and positions of most organizations in different phases will change. However, there are also a few key organizations, such as the NHCPRC, HCPCH, SCPRC, and HCHP, which have a central position in all phases of emergency communication. This shows that they assume the responsibility of connecting other organizations and have a strong influence on the information resources.

\subsection{Dynamic analysis of emergency organizations-communication tasks}

The coupling relationship between organization and task will change over time. This section mainly analyzes the Two-mode network between emergency organization and communication tasks at each phase. Figure 4 shows the constructed Two-mode network. Among them, the displayed node size is determined by the degree centrality.

As shown in Fig. 4, there are differences in the number of EIOCSTs involved in different phases. The whole emergency response process includes a total of 11 EIOCSTs, of which the number of EIOCSTs in phases P1, P2, and P3 are 5, 10, and 10, respectively. According to the analysis of the task position shown by the size of the nodes in the figure, in the early phase of the epidemic, the emergency task is mainly to organize medical treatment and epidemic prevention, and the main emergency organization is the health department. In phase P2, a large number of new organizations are engaged in new emergency tasks such as communication and transportation, logistics support, and order maintenance, and these tasks receive attention. On the contrary, in phase P3, as the epidemic is under control, many organizations withdraw, the influence of logistics support, communication and transportation and some other tasks has declined, and the resuming work and production are in a central position. The work and production resumption organization led by the State Council issued various policies to promote the resumption of work and production according to the level of the epidemic in each district. The analysis shows that in the process of emergency response, the position of a communication task will change due to the phased needs of the emergency.

Also, the number and types of the participating organizations in the same EIOCST have changed over time. In phase P2, the task of material support is mainly participated by 16 organizations including DFHP, Hubei Provincial Medical Security Bureau (HPMSB) and enterprises. In phase P3, the participants in this task changed to 8 organizations including Red Cross Society of China (RCSC), Hubei Provincial Public Security Department (HPPSD) and enterprises, indicating that the number of participating organizations in the same EIOCST has changed. Meanwhile, the medical rescue and epidemic prevention during phase P1 involved government organizations (NHCPRC, HCHP, WMHC, etc.) and research institutions (CAMS, AMMS). But in phase P2, Artificial Intelligence Diagnosis System (AIDS) and RCSC have become the new types of emergency organizations to participate in this task. Therefore, the types of participating organizations in the same EIOCST have also changed over time.

In addition to the dynamic changes in the number of EIOCSTs and the corresponding participating organizations, key EIOCSTs in different phases are analyzed. The blue rectangles in Fig. 4 represent the emergency communication tasks, and the sizes are determined by the degree centrality. The greater the degree centrality of the node, the more important the emergency communication task is. From the perspective of the entire emergency response process, communication tasks such as medical rescue, comprehensive coordination, information release, monitoring and warning, and logistical support in the epidemic 

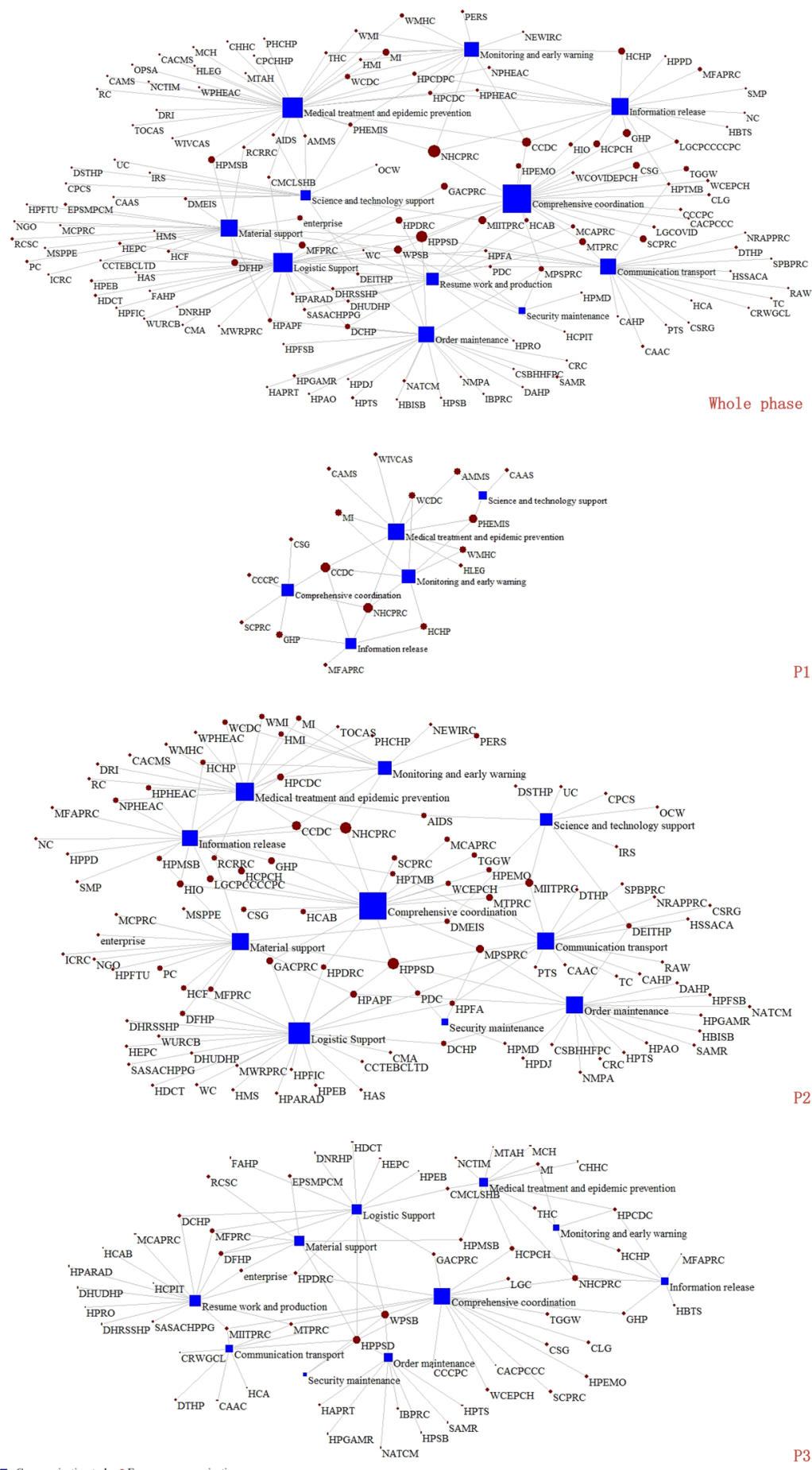

Fig. 4 Emergency organization-task two-mode network topology diagram. Note: The size of nodes set by degree centrality. The Organization's full names in Fig. 4 are listed in Table 6 of Appendix 1 
emergency response are relatively important, reflecting the goal of minimizing casualties and maintaining social stability in the epidemic response.

In order to further identify the key EIOCSTs in different phases, we calculated the degree centrality of the communication tasks involved in different phases, as shown in Fig. 5. In phase P1, the degree centrality of EIOCST4 is the largest, indicating that at phase $\mathrm{P} 1$, a large number of organizations have communicated and cooperated around the task of medical treatment and epidemic prevention. This result is consistent with actual demand at the early stage of the COVID-19 emergency, such as organizing infectious epidemiological investigations and medical rescue. In addition, monitoring and warning and comprehensive coordination are also key tasks in this phase. In phase P2, the degree centrality of EIOCST7 is greatest. With the investment of a large number of organizations and resources, emergency response has transformed from a small number of key tasks to the need for comprehensive coordination of rescue, logistics, communications and transportation, making the task of comprehensive coordination more prominent from phase P1 to phase P2. In phase P3, comprehensive coordination is still the core task, and the work resumption has become another key task for the first time due to the need for economic development. The above analysis shows that in the whole emergency process, comprehensive coordination has always been a critical task, and the main tasks of COVID-19 emergency have changed to some extent over time according to the emergency needs at different phases.

\subsection{Analysis of emergency communication frequency between different types of organizations}

Due to the large number of participating organizations and their complex interactions in the whole COVID-19 emergency process, this section analyzes the communication relationship between different levels of government organizations and between different types of organizations from the horizontal and vertical dimensions. In this analysis, we classify NAGOs, PGOs and MPOs as GOs, and other organizations as NGOs. Table 5 shows the frequency of communication between different types of organizations. Firstly, the communication within the government system is frequent, and the frequency of communication

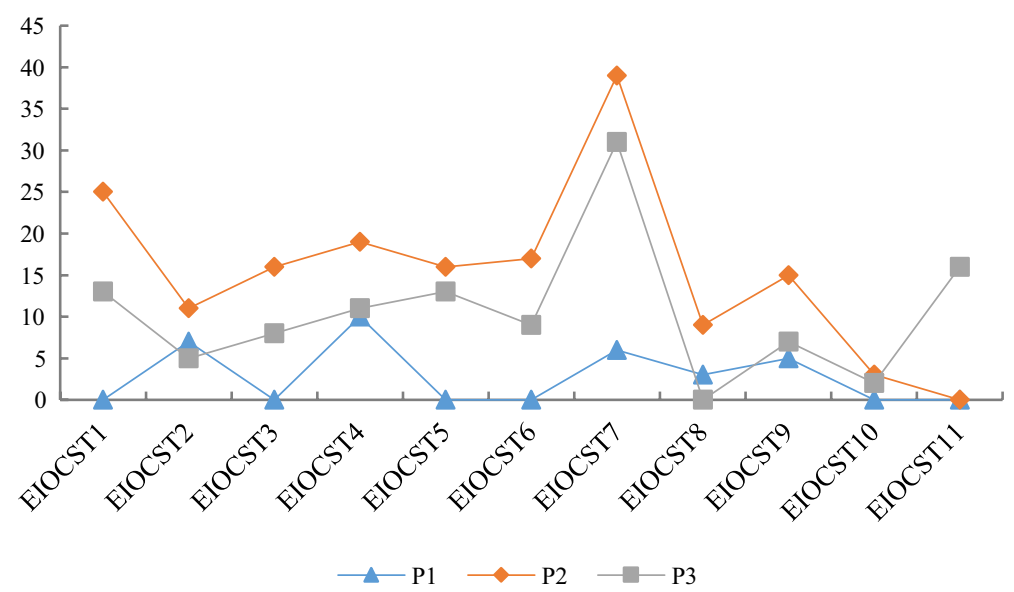

Fig. 5 Measurement of degree centrality of tasks supported by emergency communication 
Table 5 The frequency of communication between different types of emergency organizations

\begin{tabular}{llllllll}
\hline & NAGO & PGO & MGO & RI & PO & NPO & IO \\
\hline NAGO & 109 & 123 & 62 & 14 & 8 & 3 & 6 \\
PGO & - & 295 & 55 & 9 & 32 & 9 & 4 \\
MGO & - & - & 37 & 1 & 9 & 2 & 3 \\
RI & - & - & - & 6 & 8 & 0 & 2 \\
PO & - & - & - & - & 1 & 4 & 3 \\
NPO & - & - & - & - & - & 3 & 0 \\
IO & - & - & - & - & - & - & 0 \\
\hline
\end{tabular}

between GOs at the same level is more prominent, especially between PGOs. This is consistent with China's centralized management system and the level-by-level reporting system in emergency plans. Secondly, compared with the communication within the government system, the communication in NGOs and between NGOs and GOs is relatively poor. However, it is worth noting that the communication between POs and PGOs is relatively active, which may be related to the active cooperation of relevant enterprises (such as medical supplies, finance, logistics and e-commerce enterprises) in the epidemic emergency to provide material guarantees for the fight against the epidemic. In addition, research institutions also maintain certain communication with government organizations and private organizations, which is related to the cooperation and exchanges between research institutions and these organizations under normal epidemic conditions. On the whole, in the COVID-19 emergency, the communication within the government system is the most active, the communication between NGOs is relatively weak, and some NGOs maintain a certain degree of communication with GOs.

\section{Discussion}

1. Statistical analysis of indicators such as network density, network centrality, and average path length shows that COVID-19-EIOCN is dynamically evolving, and it changes from concentrated to decentralized. In the early phase of the epidemic, the emergency task is mainly to organize medical treatment and epidemic prevention, there were only a few types of participating organizations, mainly government organizations and research institutions, and interactions between organizations were frequent, so the overall network was relatively concentrated. During the outbreak phase of the epidemic, a large number of organizations joined in coordinating to handle the booming emergency tasks, such as communication and transportation, logistics support, and order maintenance, the organizations did not establish as many contacts as possible, so the overall cohesion of the network decreased. After the epidemic was controlled, the urgency of emergency response was reduced, and the withdrawal of some organizations caused related communication links to be interrupted. As a result, network cohesion was further reduced.

2. The node centrality analysis shows that in COVID-19-EIOCN, the positions and roles of organizations have changed at different phase, and the central nodes of the network are mostly composed of government organizations. The national and provincial health committees are responsible for responding to public health emergencies and providing medical rescue services, always acted as information hubs and bridges. After the 
establishment of the $\mathrm{HCPCH}$, it has become the direct commander and organizer of COVID-19 emergency activities, which was a central node and bridge organization. In addition, in the early phase of COVID-19 epidemic, government organizations and research institutions were in an important position. During the outbreak period, except the above two types of organizations, the newly added artificial intelligence systems and enterprises have also played an important role in providing medical supplies and services. In the later phase of the epidemic response, due to the needs of resuming production, emergency organizations such as HPDRC and DHRSSHP have improved their status in the network.

3. The analysis of the Two-mode network shows that the importance tasks supported by emergency organization communication change dynamically in different phases. In the early phase of COVID-19 epidemic, Medical treatment, Medical treatment and Epidemic prevention and comprehensive coordination occupy an important position. During the outbreak of the epidemic, the core emergency communication tasks are mainly logistics support, comprehensive coordination and order maintenance. In the later phase of emergency response, comprehensive coordination still occupies an important position, and the resumption of work and production has also become a new important emergency task. Therefore, the importance of tasks is related to emergency demand of major epidemics at a specific stage, which is consistent with the viewpoint of Guo et al. (2018). Thus, in response to emergency tasks in different phases, emergency communication strategies should be dynamically adjusted in time.

4. Analysis of the frequency of communication among various types of organizations shows that the emergency communication within the government system is relatively smooth. This is consistent with China's centralized management system and the levelby-level reporting system in emergency plans. In the COVID-19 emergency, the communication between NGOs is relatively weak, and the interaction between the government system and the non-governmental system is limited. These communications are mainly focused on the communication between the company and the government in material and logistics support, and the cooperation between research institutions and the government and companies in the research of testing reagents and drugs. However, for the prevention and control of major epidemics, the effective implementation of non-pharmaceutical intervention emergency activities cannot rely on a single type of organization, it requires the coordinated response of the GOs and NGOs. To reduce the pressure and risks caused by solely relying on government emergency response, the communication between the GOs and RIs, POs and NPOs should be strengthened.

In summary, because the communication between emergency organizations mainly supports the implementation of emergency tasks, the organizations need to maintain smooth communication to efficiently carry out emergency response. This article provides a new perspective for the research on emergency communication of public health emergencies from the perspective of time dynamics. The analysis found that there are dynamic changes in the tasks supported by emergency communication at different stages, and the positions and roles of participating organizations have also changed. However, there are also several core emergency organizations with a relatively stable position. They are all government organizations, which help to integrate resources and information in a short time. Since China currently implements a joint emergency response mode led by governments at several levels and supplemented by social efforts. The COVID-19 emergency response is carried out under this hierarchical centralized 
command scenario. In the early phase of the COVID-19 emergency, due to the urgency and uncertainty of the epidemic, the health administrative department efficiently organized emergency teams and integrated resources to achieve rapid coordinated response. It proves that the centralized management system is more conducive to the rapid coordination and resource integration in emergency situations, and effectively reduces the risk of poor communication between organizations. This is in line with the conclusions of Du et al. (2020) that centralized management systems may be more conducive to the integration of emergency resources and information in the shortest time under emergency situations. In addition, with the development of COVID-19, the mechanism and prevention and control methods of the epidemic have gradually become clear, the decentralized network became more conducive to inter-organizational communication and emergency operations under multi-task. This is consistent with opinion that decentralized network is more conducive to information interaction to support decision-making in the study of Hossain et al. (2015). Therefore, in the COVID-19 emergency, centralized and decentralized networks are complementary. In the actual emergency response to public health emergencies, the advantages of centralized emergency network and decentralized emergency network should be reasonably used according to the evolution of the event, and emergency communication strategies should be dynamically adjusted in phases.

\section{Conclusions}

This article studied the COVID-19-EIOCN in Wuhan, Hubei, China from the perspective of time dynamics. First, the various organizations involved in the COVID-19 emergency were identified, and then the interactions between them were extracted. Finally, the dynamic evolution of ECN, organization positions and organization communication supported tasks is analyzed through SNA.

The results of this study are helpful to guide emergency management of public health emergencies. First of all, emergency communication of public health emergencies needs to consider dynamics. The positions and tasks of emergency organizations at each stage should be clarified, information and resources should be dynamically integrated according to the evolution of specific events, and the positions and interactions of participating organizations should be dynamically adjusted to meet the emergency needs of different phases. Secondly, under the centralized management emergency system, the internal communication of the government system is relatively active, which is conducive to rapid coordination and resource integration in urgent situations, but the emergency interaction between the government system and the non-governmental system is limited. Therefore, we should promote communication between different types of organizations such as GOs, RIs, POs, and NPOs. At the same time, in the design of emergency communication structure for public 
health emergencies, it is necessary to take NGOs into consideration and set up reasonable channels for them to participate in emergency communication, so as to avoid obstructing the communication between GOs and NGOs at the system level. Finally, for future emergencies of similar public health events, we should make rational use of the advantages of centralized emergency networks and decentralized emergency networks based on the phased characteristics of event evolution, and dynamically design communication and collaboration networks to form reasonable emergency plans to meet emergency needs.

This study still has some limitations. First, the analysis of this article helps us to have a general understanding of the communication between emergency organizations under the public health emergency in China, but only a single case is used. Future research may focus on the analysis of multiple cases, such as analyzing COVID-19 ECNs at other urban, provincial and national levels. In addition, in the future, we will consider integrating network data, questionnaire surveys, interviews and other data. Questionnaires can be sent to managers of GOs and the private sector to collect data at different levels.

\section{Appendix}

See Table 6. 
Table 6 Organizations Involved in COVID-19-EIOCN

\begin{tabular}{|c|c|}
\hline Organization name & Organization abbreviation \\
\hline National Health Commission of the People's Republic of China & NHCPRC \\
\hline State Council of the PRC & SCPRC \\
\hline $\begin{array}{l}\text { Ministry of Industry and Information Technology of the People's Republic of } \\
\text { China }\end{array}$ & MIITPRC \\
\hline COVID-19 Prevention and Control System & CPCS \\
\hline Central Steering Group & CSG \\
\hline Chinese Center for Disease Prevention and Control & $\mathrm{CCDC}$ \\
\hline Drug and Medical Equipment Informantion System & DMEIS \\
\hline Civil Aviation Administration of China & CAAC \\
\hline The Online COVID-19 Assessment System & TOCAS \\
\hline Central Committee of the Communist Party of China & $\mathrm{CCCPC}$ \\
\hline Ministry of Transport of the People's Republic of China & MTPRC \\
\hline China State Railway Group Co.,Ltd & CSRG \\
\hline General Administration of Customs of the People's Republic of China & GACPRC \\
\hline Ministry of Public Security of the People's Republic of China & MPSPRC \\
\hline Ministry of Civil Affairs of the People's Republic of China & MCAPRC \\
\hline National Public Health Expert Advisory Committee & NPHEAC \\
\hline National Patriotic Health Campaign Committee & NPHCC \\
\hline $\begin{array}{l}\text { Leading Group of the CPC Central Committee for COVID-19 Prevention and } \\
\text { Control }\end{array}$ & LGCPCCCCPC \\
\hline Leading Group on COVID-19 & LGC \\
\hline National Administration of Traditional Chinese Medicine & NATCM \\
\hline Public's Electronic Reporting System & PERS \\
\hline Ministry of Foreign Affairs of the People's Republic of China & MFAPRC \\
\hline Cyberspace Administration of the CPC Central Committee & САСРССС \\
\hline State Administration for Market Regulation & SAMR \\
\hline High-Level Expert Group & HLEG \\
\hline Ministry of Finance of the People's Republic of China & MFPRC \\
\hline Central Military Commission Logistics Support and Health Bureau & CMCLSHB \\
\hline Social Welfare Home & SWH \\
\hline National Railway Administration of the People's Republic of China & NRAPPRC \\
\hline State Post Bureau of the People's Republic of China & SPBPRC \\
\hline Immigration Bureau of the People's Republic of China & IBPRC \\
\hline Public Health Emergency Monitoring Information System & PHEMIS \\
\hline National Medical Products Administration & NMPA \\
\hline National Center for Telemedicine and Internet Medicine & NCTIM \\
\hline Ministry of Education of the People's Republic of China & MEPRC \\
\hline Ministry of Water Resources of the People's Republic of China & MWRPRC \\
\hline Ministry of Commerce of the People's Republic of China & MCPRC \\
\hline $\begin{array}{l}\text { Ensuring the Production and Supply of Medical Prevention and Control Materi- } \\
\text { als }\end{array}$ & EPSMPCM \\
\hline Hubei COVID-19 Prevention and Control Headquarters & $\mathrm{HCPCH}$ \\
\hline The Government of Hubei Province & GHP \\
\hline Health Commission of Hubei Provincial & $\mathrm{HCHP}$ \\
\hline Hubei Provincial Development and Reform Commission & HPDRC \\
\hline
\end{tabular}


Table 6 (continued)

Organization name

Organization abbreviation

Hubei Province General Administration of Market Regulation

HPGAMR

Hubei Public Health Expert Advisory Committee

HPHEAC

Artificial Intelligence Resource Allocation System

AIRAS

Department of transportation of Hubei Province

DTHP

Department of Finance of Hubei Province

DFHP

Hubei Provincial Center for Disease Prevent and Control

HPCDC

Hubei Provincial Armed Police Force

HPAPF

Department of Economy and Information Technology of Hubei Province

DEITHP

Hubei Provincial Public Security Department

HPPSD

Department of Housing and Urban-rural Development of Hubei Province

DHUDHP

Hubei Safety Supervision Administration of civil aviation

HSSACA

Department of Commerce of Hubei Province

DCHP

Hubei Civil Affairs Bureau

HCAB

Department of Human Resources and Social Security of Hubei Province

DHRSSHP

Department of Science and Technology of Hubei Province

DSTHP

Hubei Department of Culture and Tourism

HDCT

Psychology Hospitals and Clinics in Hubei Province

PHCHP

Online Counselling Website

OCW

Comprehensive Supervision Bureau of Hubei Health and Family Planning

CSBHHFPC

Commission

Department of Education of Hubei Province

DEHP

Hubei Provincial Fire Administration

HPFA

Hubei provincial Traffic Management Bureau

HPTMB

Hubei provincial publicity department

HPPD

Hubei Information Office

HIO

Hubei Provincial Emergency Management Office

HPEMO

Hubei Administration of Sport

HAS

Hubei Provincial Energy Bureau

HPEB

State-Owned Assets Supervision and Administration Commission of Hubei

SASACHPPG

Province People's Government

Hubei Provincial Tax Service

HPTS

Department of Ecology and Environment of Hubei Province

DEEHP

Hubei Provincial Audit Office

HPAO

Hubei Province Agriculture and Rural Affairs Department

HPARAD

Medical Teams to Assist Hubei

MTAH

Hubei Provincial Medical Security Bureau

HPMSB

Hubei Poverty Relief Office

HPRO

Hubei Communication Administration

HCA

Department of Natural Resources of Hubei Province

DNRHP

drug administration of Hubei Province

DAHP

Hubei Electric Power Company

HEPC

Hubei Administration of Public Resource Trade

HAPRT

Cyberspace Administration of Hubei Province

CAHP

Hubei Council for the Promotion of International Trade

HCPIT

Hubei Provincial Statistics Bureau

HPSB 
Table 6 (continued)

Organization name

Organization abbreviation

Hubei Charity Federation

$\mathrm{HCF}$

Hubei Bank Insurance Supervision Bureau

HBISB

Hubei Meteorological Service

HMS

Hubei Provincial Federation of Trade Unions

HPFTU

Hubei Provincial Military Distrisct

HPMD

Hubei Provincial Financial Supervision Bureau

HPFSB

Hubei Broadcasting and Television Station

HBTS

Food Administration of Hubei Province

FAHP

Artificial Intelligence diagnosis system

AIDS

Medical institution

MI

Citizens

Citizens

Acommunity Residents Committee

CRC

Railway Administration of Wuhan

RAW

Wuhan Municipal Health Commission

WMHC

Wuhan Center for Disease Prevention and Control (CDC)

WCDC

Wuhan COVID-19 Epidemic Prevention and Control Headquarters

WCEPCH

Community

community

Wuhan Public Health Expert Advisory Committee

WPHEAC

Mobile Cabin Hospitals

$\mathrm{MCH}$

The Government of Wuhan

TGGW

Wuhan Customs

WC

Hubei Wuhan Women's Prison

HWWP

Wuhan Housing Security and Management bureau

WHSMB

Mayor Hotline 12,345

MH1

Online Psychological Counseling

OPC

China Railway Wuhan Group Co.Ltd

CRWGCL

Wuhan Public Security Bureau

WPSB

Township Health Centers

THC

Community Health Service Centers

$\mathrm{CHHC}$

Universities and Colleges

$\mathrm{UC}$

Research Centers

$\mathrm{RC}$

Domestic Research Institutions

DRI

Academy of Military Medical Sciences

AMMS

Chinese Academy of Medical Sciences

CAMS

Wuhan Institute of Virology, Chinese Academy of Sciences

WIVCAS

China Academy of Chinese Medical Sciences

CACMS

Chinese Academy of Sciences

CAAS

Red Cross society of China Hubei branch

RCRRC

International Committee of the Red Cross

ICRC

Red Cross Society of China

RCSC

Non-government Organizations

NGO

Parcel Delivery Companies

PDC

Pharmaceutical companies

$\mathrm{PC}$

Social Media Platforms

SMP 
Table 6 (continued)

\begin{tabular}{ll}
\hline Organization name & Organization abbreviation \\
\hline Enterprise & Enterprise \\
Manufacturers and Suppliers of Personal Protective Equipment (PPE) & MSPPE \\
News Companies & NC \\
Taxi Companies & TC \\
Public Transport System & PTS \\
International research institutions & IRS \\
World Health Organization & WHO \\
US Centers for Disease Control and Prevention & CDCP
\end{tabular}

Acknowledgements This study was financially supported by "safety Risk Assessment Method of Consumer products": National Key Research and Development Project (2017YFF0209604-2)

\section{Declarations}

Conflict of interest The authors declare that they have no conflict of interest.

\section{References}

Abbasi A (2014) Link formation pattern during emergency response network dynamics. Nat Hazards 71(3):1957-1969

Abbasi A, Kapucu N (2012) Structural dynamics of organizations during the evolution of interorganizational networks in disaster response. J Homel Secur Emerg Manag 9(1):1-19

Abbasi A, Kapucu N (2016) A longitudinal study of evolving networks in response to natural disaster. Comput Math Organ Theory 22(1):47-70

Abbasi A, Sadeghi-Niaraki A, Jalili M, Choi SM (2018) Enhancing response coordination through the assessment of response network structural dynamics. PLoS One 13(2):1-17

Adiyoso W (2020) Assessing Governments' Emergency Responses to COVID-19 Outbreak Using a Social Network Analysis (SNA). Advance, Preprint

Alkhatib M, El Barachi M, Shaalan K (2019) An Arabic social media based framework for incidents and events monitoring in smart cities. J Clean Prod 220(MAY 20):771-785

CDC (2020) Coronavirus Disease 2019 (COVID-19). Centers for Disease Control and Prevention

Clark-Ginsberg A (2020) Disaster risk reduction is not 'everyone's business': Evidence from three countries. Int J Disaster Risk Reduct 43

Comfort LK, Waugh WL, Cigler BA (2012) Emergency management research and practice in public administration: emergence, evolution, expansion, and future directions. Public Adm Rev 72(4):539-547

Curtis CA (2018) Organizational networks in times of crisis: lessons from Katrina. J Conting Cris Manag 26(2):202-211

Du L, Feng Y, Tang LY et al (2020) Time dynamics of emergency response network for hazardous chemical accidents: a case study in China. J Clean Prod 248:119239

Freeman LC (1978) Centrality in social networks conceptual clarification. Soc Netw 1(3):215-239

Guo X, Kapucu N (2015) Examining collaborative disaster response in China: network perspectives. Nat Hazards 79(3):1773-1789

Guo X, Zhao H, Jia S (2018) Interorganizational collaboration in emergency response based on time dynamic network. J Shanghai Adm Inst 19(06):31-44

Harris JL, Doerfel ML (2017) Interorganizational resilience: networked collaborations in communities after superstorm sandy, pp 75-91

Hossain L, Hamra J, Wigand RT, Carlsson S (2015) Exponential random graph modeling of emergency collaboration networks. Knowl-Based Syst 77:68-79 
Howes M, Tangney P, Reis K et al (2015) Towards networked governance: improving interagency communication and collaboration for disaster risk management and climate change adaptation in Australia. J Environ Plan Manag 58(5):757-776

Htein MK, Lim S, Zaw TN (2018) The evolution of collaborative networks towards more polycentric disaster responses between the 2015 and 2016 Myanmar floods. Int J Disaster Risk Reduct 31(May):964-982

Hu Q, Kapucu N (2016) Information communication technology utilization for effective emergency management networks. Public Manag Rev 18(3):323-348

Hu Q, Knox CC, Kapucu N (2014) What have we learned since september 11, 2001? A network study of the boston marathon bombings response. Public Adm Rev 74(6):698-712

Jung K, Park HW (2016) Tracing interorganizational information networks during emergency response period: a webometric approach to the 2012 Gumi chemical spill in South Korea. Gov Inf Q 33(1):133-141

Kapucu N, Garayev V (2016) Structure and network performance: horizontal and vertical networks in emergency management. Adm Soc 48(8):931-961

Kapucu N, Hu Q (2016) Understanding multiplexity of collaborative emergency management networks. Am Rev Public Adm 46(4):399-417

Kim J, Hastak M (2018) Social network analysis: characteristics of online social networks after a disaster. Int J Inf Manag 38(1):86-96

Kim KD, Hossain L, Uddin S (2013) Situated response and learning of distributed bushfire coordinating teams. J Homel Secur Emerg Manag 10(1):1-17

Koliba CJ, Meek JW, Zia A, Mills RW (2018) Governance Networks in Public Administration and Public Policy. CRC Press, Inc., London

Lai S, Ruktanonchai NW, Zhou L et al (2020) Effect of non-pharmaceutical interventions to contain COVID-19 in China. Nature 585(7825):410-413

Lim S, Nakazato H (2020) The emergence of risk communication networks and the development of citizen health-related behaviors during the COVID-19 pandemic: Social selection and contagion processes. Int J Environ Res Public Health 17(11):1-12

Liu J, Hao J, Shi Z, Bao HXH (2020) Building the COVID-19 collaborative emergency network: a case study of COVID-19 outbreak in Hubei Province, China. Nat Hazards 104(3):2687-2717

Opdyke A, Lepropre F, Javernick-Will A, Koschmann M (2017) Inter-organizational resource coordination in post-disaster infrastructure recovery. Constr Manag Econ 35(8-9):514-530

Pasman HJ, Suter G (2005) EFCE working party on loss prevention and safety promotion in the process industries. Process Saf Environ Prot 83(B1):18-21

Provan KG, Lemaire RH (2012) Core concepts and key ideas for understanding public sector organizational networks: using research to inform scholarship and practice. Public Adm Rev 72(5):638-648

Rajput AA, Li Q, Zhang C, Mostafavi A (2020) Temporal network analysis of inter-organizational communications on social media during disasters: A study of Hurricane Harvey in Houston. Int J Disaster Risk Reduct 46(April):101622

Robinson SE (2012) Brokerage and closure in emergency management networks. Zhurnal Eksp I Klin Meditsiny 15(4):32-39

Scott J (2013) Social network analysis: a handbook, 3rd edn. Sage Publications, Los Angeles

Scott J (2017) Social network analysis, 4th edn. Sage, Thousand Oaks

Tang P, Deng C, Shao S, Shen GQ (2017) Leveraging intergovernmental and cross-sectoral networks to manage nuclear power plant accidents: a case study from China. J Clean Prod 162(sep.20):1551-1566

Wen L, Kang W, Gu JY (2011) Research on emergency information management based on the social network analysis. In: Proceedings of international conference on information systems for crisis response and management, ISCRAM 2011, pp 187-192

WHO (2020a) Coronavirus disease (COVID-2019) situation reports

WHO (2020b) WHO director-general's opening remarks at the media briefing on COVID-19-11 March 2020. World Health Organization

Wolbers J, Groenewegen P, Mollee J, Bím J (2013) Incorporating time dynamics in the analysis of social networks in emergency management. J Homel Secur Emerg Manag 10(2):555-585

Publisher's Note Springer Nature remains neutral with regard to jurisdictional claims in published maps and institutional affiliations 